\title{
A DIRICHLET SERIES EXPANSION FOR THE $p$-ADIC ZETA-FUNCTION
}

\author{
DANIEL DELBOURGO
}

(Received 29 June 2004; revised 21 May 2005)

\author{
Communicated by W. W. L. Chen
}

\begin{abstract}
We prove that the $p$-adic zeta-function constructed by Kubota and Leopoldt has the Dirichlet series expansion

$$
\zeta_{p}\left(k, \omega^{1-k}\right)=\frac{1}{\left(2-4 \cdot 2^{-k}\right)} \sum_{n=1}^{\infty} \sum_{\substack{m=p^{n-1} \\ p \nmid m}}^{p^{n}} \frac{(-1)^{m+1}}{m^{k}} \text { at all } k \in \mathbb{Z},
$$

where the convergence of the first summation is for the $p$-adic topology. The proof of this formula relates the values of $\zeta_{p}\left(-s, \omega^{1+\beta}\right)$ for $s \in \mathbb{Z}_{p}$, with a branch of the ' $s^{\text {th }}$-fractional derivative' of a suitable generating function.
\end{abstract}

2000 Mathematics subject classification: primary 11R23, 11G55, 11 R60.

\section{Statement of results}

Ever since its discovery over fifty years ago, the $p$-adic Riemann zeta-function has long been considered a very different animal to its complex cousin. Despite sharing their special values at negative integers, the two exhibit vastly different behaviours. The purpose of this note is to show that as analytic functions, they are not as dissimilar as they first appear to the naked eye.

Fix an odd rational prime number $p$, and let us write $\mathbb{C}_{p}=\hat{\overline{\mathbb{Q}}}_{p}$ for the Tate field. For any $x \in \mathbb{Z}_{p}^{\times}$, there is a natural decomposition $x=\omega(x)\langle x\rangle$, where $\omega$ is the Teichmüller character modulo $p$, and $\langle x\rangle$ denotes the projection to the principal units $1+p \mathbb{Z}_{p}$. Kubota and Leopoldt defined a $p$-adic $L$-function $\zeta_{p}(s,-)$ over the space

(C) 2006 Australian Mathematical Society $1446-7887 / 06 \$ \mathrm{~A} 2.00+0.00$ 
$\left\{s \in \mathbb{C}_{p}:|s|_{p}<p^{(p-2) /(p-1)}\right\}$, satisfying the interpolation rule

$$
\zeta_{p}\left(1-n, \omega^{n}\right)=\left(1-p^{n-1}\right) \zeta_{\infty}(1-n) \quad \text { for every integer } n \geq 1 .
$$

At negative integers the complex Riemann zeta-function will take rational values $\zeta_{\infty}(1-n)=-B_{n} / n$ where $B_{n}$ is the $n$-th Bernoulli number, so the right-hand side above is viewed as a $p$-adic number. For a fixed congruence class $\beta$ modulo $p-1$, the branches $\zeta_{p}\left(s, \omega^{\beta}\right)$ are all analytic functions, except for a simple pole at $s=1$ when $\beta \equiv 0(\bmod p-1)$.

There are several different ways to construct these objects. One method is to take a $p$-adic Mellin transform of the generalised Bernoulli distribution. Another approach is the theory of Coleman power series, which converts cyclotomic units into $p$-adic $L$-functions. The following result shows there is yet a third construction, strongly reminiscent of the Dirichlet series $\sum_{n=1}^{\infty} 1 / n^{z}$, defining the standard Riemann zeta-function at points $z \in \mathbb{C}, \operatorname{Re}(z)>1$.

THEOREM 1.1. For all $s \in \mathbb{Z}_{p}$ and branches $\beta$ modulo $p-1$,

$$
\zeta_{p}\left(-s, \omega^{1+\beta}\right)=\frac{1}{2\left(1-\omega^{1+\beta}(2)\langle 2\rangle^{1+s}\right)} \sum_{n=1}^{\infty}{ }_{\beta} \Delta(n, s),
$$

where convergence takes place in $\mathbb{Q}_{p}$, and the ${ }_{\beta} \Delta(-,-)$ 's are defined by

$$
{ }_{\beta} \Delta(n, s):=\sum_{\substack{m=p^{n-1} \\ p \nmid m}}^{p^{n}}(-1)^{m+1} \omega^{\beta}(m)\langle m\rangle^{s} .
$$

The $p$-adic power $\langle m\rangle^{s}=\exp (s \log m)$ makes sense only if $p \nmid m$, and is properly defined for $|s|_{p}<p^{(p-2) /(p-1)}$. In due course we shall prove that the $p$-adic sequence $\left\{_{\beta} \Delta(n, s)\right\}_{n \in \mathbb{N}}$ tends to zero at the rate $O\left(p^{-n}\right)$.

This seems to be the first instance where a $p$-adic $L$-function has been expanded as a Dirichlet series. Unfortunately, it does not seem feasible to go a stage further and express the summation as an Euler product. The formal identity

$$
\sum_{n=1}^{\infty} n^{-s}=\frac{1}{\left(1-l^{-s}\right)} \sum_{n \in \mathbb{N}-l \mathbb{N}} n^{-s} \text { for a prime number } l \neq p
$$

has no meaningful convergence in the rigid analytic topology. For similar reasons, the Euler factor $\left(1-\omega^{1+\beta}(2)\langle 2\rangle^{1+s}\right)$ cannot be absorbed into $\sum_{n=1}^{\infty} \Delta(n, s)$.

Let us now check what happens when we assume that $s=-k$ is a rational integer. Choosing the class $\beta \equiv-k(\bmod p-1)$ means that the term $\omega^{\beta}(m)\langle m\rangle^{s}=m^{-k}$, from which we deduce ${ }_{\beta} \Delta(n,-k)=\sum_{m=p^{n-1}, p \nmid m}^{p^{n}}(-1)^{m+1} m^{-k}$. 
COROLlaRY 1.2. If $k \in \mathbb{Z}$ is an integer, then

$$
\zeta_{p}\left(k, \omega^{1-k}\right)=\frac{1}{2\left(1-2^{1-k}\right)} \sum_{n=1}^{\infty}\left(\sum_{\substack{m=p^{n-1} \\ m \text { odd, } p \nmid m}}^{p^{n}} \frac{1}{m^{k}}-\sum_{\substack{m=p^{n-1} \\ m \text { even, } p \nmid m}}^{p^{n}} \frac{1}{m^{k}}\right),
$$

where the first summation converges inside of $\mathbb{Q}_{p}$.

At the exceptional point $k=1$, the $p$-adic zeta-function has a simple pole. This is reflected on the right-hand side by a zero in the Euler factor $\left(1-2^{1-k}\right)$.

If $k \leq 0$, the left-hand side equals $\left(1-p^{-k}\right) \zeta_{\infty}(k)=-\left(1-p^{-k}\right) B_{1-k} /(1-k)$, which means the Bernoulli numbers can be expressed as a $p$-adic Dirichlet series. In fact, our calculations produce the congruence

$$
\left(1-2^{1-k}\right)\left(1-p^{-k}\right) \times \frac{B_{1-k}}{1-k} \equiv-\frac{1}{2} \sum_{m=1, p \nmid m}^{p^{n}} \frac{(-1)^{m+1}}{m^{k}} \quad\left(\bmod p^{n}\right)
$$

at all integers $k \leq 0$ and $n \geq 1$, which we could not find anywhere in the literature.

On the other hand, if $k \geq 2$, then $\zeta_{p}\left(k, \omega^{1-k}\right)$ no longer interpolates the classical Riemann zeta-function. However, Coleman [2] has proven the limit equation

$$
\left(1-p^{-k}\right) \lim _{x \rightarrow 1}\left(\ell_{k}(x)-p^{-k} \ell_{k}\left(x^{p}\right)\right)=\zeta_{p}\left(k, \omega^{1-k}\right),
$$

where $\ell_{k}(x)=\sum_{m=1}^{\infty} x^{m} / m^{k}$ is the polylogarithm, and $x$ lies in $\mathbb{P}^{1}\left(\mathbb{C}_{p}\right)-\{0,1, \infty\}$. In particular, combining his theorem with Corollary 1.2 yields an explicit expression for the $p$-adic polylogarithm. We do not know whether this formula can be obtained directly using rigid analysis.

REMARK 1.3. A curious phenomenon occurs when $k$ is an integer greater than one. Not only do the terms

$$
{ }_{-k} \Delta(n,-k)=\sum_{\substack{m=p^{n-1} \\ m \text { odd, } p \nmid m}}^{p^{n}} \frac{1}{m^{k}}-\sum_{\substack{m=p^{n-1} \\ m \text { even, } p \nmid m}}^{p^{n}} \frac{1}{m^{k}}
$$

tend to zero in the $p$-adic topology, but they also do for the archimedean topology. An elementary computation involving partial sums reveals that

$$
\sum_{n=1}^{\infty}{ }_{-k} \Delta(n,-k)=\left(1-2^{1-k}\right)\left(1-p^{-k}\right) \times \zeta_{\infty}(k),
$$

this time the convergence being inside $\mathbb{R}$. Thus for any integer $N \gg 1$, the rational number $\sum_{n=1-k}^{N} \Delta(n,-k)$ simultaneously approximates $\zeta_{p}\left(k, \omega^{1-k}\right) p$-adically, and approximates $\zeta_{\infty}(k)$ as a real number! 


\section{Fractional logarithmic differentiation}

The fractional calculus was first invented by Liouville in the nineteenth century. In a nutshell, it assigns a meaning to the operator $d^{n} / d X^{n}$ when the order $n \notin \mathbb{Z}$. It has since turned into a very valuable tool in the control of dynamical systems. Vladimirov [3] introduced the $p$-adic fractional derivative within the context of mathematical physics, where it has applications to wavelet analysis and symmetry-breaking.

Our expansion for the $p$-adic zeta-function is based on the observation that CoatesWiles homomorphisms are, in some sense, fractional derivations in disguise. Let $K$ be a finite extension of $\mathbb{Q}_{p}$, with ring of integers $\mathscr{O}$. The topological power series ring $\Lambda=\mathscr{O}[[X]]$ is often referred to as the Iwasawa algebra. For all $n \in \mathbb{N}$, we shall write $P_{n}(X)$ for the polynomial $(1+X)^{p^{n}}-1$. There is a well-known isomorphism

$$
\Lambda \cong{\underset{\leftarrow}{n}}_{\lim } \mathscr{O}[X] / P_{n} \mathscr{O}[X]
$$

which is described in [4, Section 7.1].

The algebra $\Lambda$ possesses a useful division algorithm. If $F(X) \in \Lambda$ and $n \geq 1$, then there exist unique elements $Q_{n}(X) \in \Lambda$ and $R_{n}(X) \in \mathscr{O}[X]$ such that

$$
F(X)=P_{n}(X) Q_{n}(X)+R_{n}(X) \text { with } \operatorname{deg}\left(R_{n}\right)<p^{n} .
$$

The $R_{n}$ 's form a Cauchy sequence in the topology of $\Lambda$, tending to $F$ as $n \rightarrow \infty$.

LEMMA 2.1. If we set

$$
\Theta_{n}(X):=p^{-n}\left(\frac{X^{p^{n}}-1}{X-1}\right) \in \mathbb{Q}[X]
$$

then

$$
R_{n}(X)=\sum_{\alpha \in \mu_{p^{n}}} F\left(\alpha^{-1}-1\right) \Theta_{n}(\alpha(1+X)),
$$

where $\mu_{p^{n}}$ denotes the group of $p^{n}$-th roots of unity.

PROOF. As both sides are polynomials of degree $<p^{n}$, it is enough to show that they agree at $p^{n}$ distinct points. If $\xi$ is any $p^{n}$-th root of unity, then the right-hand side evaluated at $X=\xi-1$ equals

$$
\sum_{\alpha \in \mu_{p^{n}}} F\left(\alpha^{-1}-1\right) \Theta_{n}(\alpha \xi)=F(\xi-1) \Theta_{n}(1)+\sum_{\alpha \neq \xi^{-1}} F\left(\alpha^{-1}-1\right) \times 0,
$$

since $\Theta_{n}$ vanishes at non-trivial $p^{n}$-th roots of unity.

In fact $\Theta_{n}(1)=1$ and $F(\xi-1)=R_{n}(\xi-1)$, so both polynomials coincide on the set of values $\left\{\xi-1: \xi \in \mu_{p^{n}}\right\}$. The result follows. 
Consider the $\mathscr{O}$-linear differential operator $(1+X)(d / d X): \Lambda \rightarrow \Lambda$ introduced in [1]. If we put $X=\exp (Z)-1$ so that $F(\exp (Z)-1) \in K$ [[Z]], then

$$
\frac{d^{k}}{d Z^{k}} F(\exp (Z)-1)=\left((1+X) \frac{d}{d X}\right)^{k} \circ F(X),
$$

which justifies the terminology logarithmic derivative.

Note that $(1+X)(d / d X)$ is not invertible on the whole of $\Lambda$. However, defining the idempotent $\psi$ by

$$
\psi F(X):=F(X)-\frac{1}{p} \sum_{\xi \in \mu_{p}} F(\xi(1+X)-1),
$$

it can be shown that $(1+X)(d / d X): \Lambda^{\psi=1} \rightarrow \Lambda^{\psi=1}$ is a bijective derivation.

For example, if we take a polynomial $G(X)=\sum_{m=0}^{\operatorname{deg}(G)} g_{m}(1+X)^{m} \in \mathscr{O}[X]$, then

$$
\psi G(X)=\sum_{m=0, p \nmid m}^{\operatorname{deg}(G)} g_{m}(1+X)^{m},
$$

so $\psi$ kills off terms of the form $(1+X)^{m p}$.

LEMMA 2.2. If $F, P_{n}, Q_{n}$ and $R_{n}$ are as above, then for all $k \in \mathbb{N}$

$$
\left((1+X) \frac{d}{d X}\right)^{k} \circ \psi F(X) \equiv\left((1+X) \frac{d}{d X}\right)^{k} \circ \psi R_{n}(X) \quad\left(\bmod (p, X)^{n}\right) .
$$

PROOF. A bare-hands calculation shows that

$$
\begin{aligned}
((1+ & \left.X) \frac{d}{d X}\right) \circ \psi\left(P_{n}(X) Q_{n}(X)\right) \\
& =\left((1+X) \frac{d}{d X}\right) \circ\left(P_{n}(X) . \psi Q_{n}(X)\right) \\
& =P_{n}(X)(1+X) \frac{d}{d X} \psi Q_{n}(X)+p^{n}(1+X)^{p^{n}} \psi Q_{n}(X),
\end{aligned}
$$

which lies in $P_{n} \Lambda+p^{n} \Lambda \subset(p, X)^{n}$, since $P_{n} \in(p, X)^{n+1}$.

By using induction on $k$, the congruence is easily established.

For a fixed congruence class $\beta(\bmod p-1)$, we shall write $\mathscr{S}_{\beta}$ for the set of positive integers congruent to $\beta$ modulo $p-1$. On our polynomial $G(X) \in \mathscr{O}[X]$,

$$
\left((1+X) \frac{d}{d X}\right)^{k} \circ G(X)=\sum_{m=0}^{\operatorname{deg}(G)} m^{k} \times g_{m}(1+X)^{m} .
$$


If $p$ does not divide $m$, then $m^{k}$ is a continuous function of $k \in \mathscr{S}_{\beta}$, in fact

$$
m^{k}=\omega^{k}(m)\langle m\rangle^{k}=\omega^{\beta}(m) \times \exp (k \log m) .
$$

However, if $p$ divides $m$ then $m^{k}$ tends to zero as $k \rightarrow \infty$, which indicates we must first cut out by $\psi$ to obtain continuity.

DEFINITION 2.3. For a class $\beta(\bmod p-1)$ and any $s \in \mathbb{Z}_{p}$, we define

$$
{ }_{\beta} D^{s}: \mathscr{O}[X]^{\psi=1} \rightarrow \mathscr{O}[X]^{\psi=1} \quad \text { to be the limit }{ }_{\beta} D^{s}=\lim _{\substack{k \rightarrow s \\ k \in \mathcal{S}_{\beta}}}\left((1+X) \frac{d}{d X}\right)^{k} .
$$

This is unique and well-defined, since each set $\mathscr{S}_{\beta}$ is clearly dense in $\mathbb{Z}_{p}$. Moreover, its action on the polynomial $\psi G(X) \in \mathscr{O}[X]^{\psi=1}$ is given by the simple formula

$$
{ }_{\beta} D^{s} \circ \psi G(X)=\sum_{m=0, p \nmid m}^{\operatorname{deg}(G)} \omega^{\beta}(m)\langle m\rangle^{s} g_{m}(1+X)^{m} \quad \text { for all } s \in \mathbb{Z}_{p} .
$$

Bearing in mind the approximation in Lemma 2.2, and upon observing that the ideals $(p, X)^{n}$ form a decreasing sequence of neighborhoods of zero, we have shown the following.

PROPOSITION 2.4. For any $F(X) \in \Lambda$ with convergents $\left\{R_{n}(X)\right\}_{n \in \mathbb{N}}$, the limit ${ }_{\beta} D^{s} \circ \psi F(X):=\lim _{n \rightarrow \infty}\left({ }_{\beta} D^{s} \circ \psi R_{n}(X)\right)$ exists and is well defined. For each congruence class $\beta$ modulo $p-1$, it follows that ${ }_{\beta} D^{s}: \Lambda^{\psi=1} \rightarrow \Lambda^{\psi=1}$ is the unique extension to the Iwasawa algebra of the operator given in Definition 2.3.

The operator ${ }_{(-)} D^{s}$ has $p-1$ branches, just like the $p$-adic $L$-function. The following properties of ${ }_{\beta} D^{s}$ (in particular property (iv)) illustrate why the terminology fractional logarithmic derivative is appropriate.

LEMMA 2.5. (i) For $\beta_{1}, \beta_{2} \in \mathbb{Z} /(p-1) \mathbb{Z}$ and $s_{1}, s_{2} \in \mathbb{Z}_{p}$, we have ${ }_{\beta_{1}} D^{s_{1}} \circ$ ${ }_{\beta_{2}} D^{s_{2}}={ }_{\beta_{1}+\beta_{2}} D^{s_{1}+s_{2}}$;

(ii) If $k \in \mathscr{S}_{\beta}$, then ${ }_{\beta} D^{k}=((1+X) d / d X)^{k}$;

(iii) For all $s \in \mathbb{Z}_{p}$, the p-fold composition ${ }_{\beta} D^{s} \circ \cdots{ }_{\beta} D^{s}={ }_{\beta} D^{p s}$;

(iv) If $a, b \in \mathbb{N}$ such that $\operatorname{gcd}(b, p(p-1))=1$, and $\beta \equiv a b^{-1}(\bmod p-1)$, then the $b$-fold composition ${ }_{\beta} D^{a / b} \circ \cdots \circ_{\beta} D^{a / b}=((1+X) d / d X)^{a}$.

Due to the density of the subring of polynomials inside $\Lambda$, it is enough to check these statements (i)-(iv) on elements of $\mathscr{O}[X]^{\psi=1}$. We leave them as an easy exercise for the reader. 


\section{Estimating the zeta-function}

Let us return to the task of calculating the Dirichlet series for the Kubota-Leopoldt zeta-function. We need two things: a formula relating fractional derivatives with $L$-functions, and a sequence of good numerical approximations to the fractional derivative. We start by addressing the latter problem.

Again $F(X) \in \Lambda$ with convergent polynomials $\left\{R_{n}(X)\right\}_{n \in \mathbb{N}}$. Recall that if the positive integer $n$ is very large, then the ideal $(p, X)^{n}$ is very small (topologically).

LEMMA 3.1. For all integers $n \geq 1$, we have the approximation modulo $(p, X)^{n}$

$$
{ }_{\beta} D^{s} \circ \psi F(X) \equiv \sum_{m=1, p \nmid m}^{p^{n}} \omega^{\beta}(m)\langle m\rangle^{s}\left(\frac{\sum_{\alpha \in \mu_{p^{n}}} \alpha^{m} F\left(\alpha^{-1}-1\right)}{p^{n}}\right)(1+X)^{m} .
$$

Proof. From Lemmas 2.2 and 2.5 (ii), we know that ${ }_{\beta} D^{s} \circ \psi F(X) \equiv{ }_{\beta} D^{s} \circ \psi R_{n}(X)$ modulo $(p, X)^{n}$ whenever $s \in \mathscr{S}_{\beta}$. Indeed, the continuity built into the fractional derivative ensures this congruence holds true for all $s \in \mathbb{Z}_{p}$.

As a consequence, it is enough to prove that ${ }_{\beta} D^{s} \circ \psi R_{n}(X)$ equals the right-hand side of the above equation. By a direct application of Lemma 2.1,

$$
R_{n}(X)=\sum_{\alpha \in \mu_{p^{n}}} F\left(\alpha^{-1}-1\right) p^{-n} \sum_{m=0}^{p^{n}-1} \alpha^{m}(1+X)^{m}
$$

whence

$$
{ }_{\beta} D^{s} \circ \psi R_{n}(X)=\sum_{\alpha \in \mu_{p^{n}}} F\left(\alpha^{-1}-1\right) p^{-n} \sum_{m=0, p \nmid m}^{p^{n}-1} \omega^{\beta}(m)\langle m\rangle^{s} \alpha^{m}(1+X)^{m} .
$$

This expression is equivalent to the formula in the statement of the lemma.

PROPOSITION 3.2. If $\mathscr{L}_{2}(X):=(X+2)^{-1}$ then for all $s \in \mathbb{Z}_{p}$ and $\beta(\bmod p-1)$,

$$
\frac{{ }_{\beta} D^{s} \circ \psi \mathscr{L}_{2}(0)}{\left(1-\omega^{1+\beta}(2)\langle 2\rangle^{1+s}\right)}=-\zeta_{p}\left(-s, \omega^{1+\beta}\right)
$$

PROOF. We first remark that $\mathscr{L}_{2}(X)$ is a power series with $p$-integral coefficients, convergent everywhere on $\mathbb{Z}_{p}$ except $X=-2$; in particular, it certainly lies in $\Lambda$. Furthermore, we can rewrite it in the form

$$
\mathscr{L}_{2}(X)=\frac{1}{X}-\frac{2}{(1+X)^{2}-1},
$$


and then changing variable yields the formal identity

$$
\mathscr{L}_{2}(\exp (Z)-1)=\frac{1}{Z}\left(\frac{Z}{\exp (Z)-1}-\frac{2 Z}{\exp (2 Z)-1}\right) .
$$

Recall that the power series expansion $Z /(\exp (Z)-1)=\sum_{n=0}^{\infty} B_{n} Z^{n} / n$ ! gives birth to the Bernouilli numbers $B_{n}$.

We make the important observation that for all $k \in \mathbb{N}$,

$$
\begin{aligned}
\left.\left((1+X) \frac{d}{d X}\right)^{k} \psi \mathscr{L}_{2}(X)\right|_{X=0} & =\left.\left(\frac{d}{d Z}\right)^{k} \psi \mathscr{L}_{2}(\exp (Z)-1)\right|_{Z=0} \\
& =\left(1-2^{1+k}\right)\left(1-p^{k}\right) \frac{B_{1+k}}{1+k} .
\end{aligned}
$$

Actually this is a well-known formula found by Coates and Wiles [1], although an analogous result can be traced back more than 150 years to the work of Kummer. We do not intend to reproduce the details here, and instead refer the reader to the description in [4, Section 12.2].

The natural numbers $\mathbb{N}$ are the disjoint union of the sets $\mathscr{S}_{\beta}$ with $\beta \in \mathbb{Z} /(p-1) \mathbb{Z}$. For all integers $k \in \mathscr{S}_{\beta}$, the values $\left.((1+X) d / d X)^{k} \psi \mathscr{L}_{2}(X)\right|_{X=0}$ are continuously interpolated by the function ${ }_{\beta} D^{s} \circ \psi \mathscr{L}_{2}(0)$ at points $s \in \mathbb{Z}_{p}$. Similarly, the Euler factor $\left(1-2^{1+k}\right)$ is interpolated by $\left(1-\omega^{1+\beta}(2)\langle 2\rangle^{1+s}\right)$ in a $p$-adic sense. Lastly, the critical values $\left(1-p^{k}\right) B_{1+k} /(1+k)$ are precisely those of minus the Kubota and Leopoldt $p$-adic $L$-function, evaluated at negative integers.

It is time to combine these strands together, and give the proof of Theorem 1.1. Let $\beta$ denote a branch modulo $p-1$, and let $s$ be any $p$-adic integer. Then

$$
\begin{aligned}
\left(1-\omega^{1+\beta}(2)\langle 2\rangle^{1+s}\right) \zeta_{p}\left(-s, \omega^{1+\beta}\right) & \stackrel{\text { by } 3.2}{=}-{ }_{\beta} D^{s} \circ \psi \mathscr{L}_{2}(0) \\
& \stackrel{\text { by } 3.1}{\equiv}-\sum_{m=1, p \nmid m}^{p^{n}} \omega^{\beta}(m)\langle m\rangle^{s} \Omega_{m}\left(\mathscr{L}_{2}\right) \quad\left(\bmod p^{n}\right),
\end{aligned}
$$

where the symbol $\Omega_{m}\left(\mathscr{L}_{2}\right)$ denotes the summation $p^{-n} \sum_{\alpha \in \mu_{p^{n}}} \alpha^{m} \mathscr{L}_{2}\left(\alpha^{-1}-1\right)$.

KeY ClaIM. If $0 \leq m \leq p^{n}-1$, then $\Omega_{m}\left(\mathscr{L}_{2}\right)=(-1)^{m} / 2$.

Certainly if this assertion is correct, then Theorem 1.1 follows immediately from it. In fact not only do the summands ${ }_{\beta} \Delta(n, s)$ of the Introduction tend to zero as $n \rightarrow \infty$, but they do so at exactly the rate $O\left(p^{-n}\right)$.

PROOF. To establish the truth of our Key Claim, we use simple induction on $m$. Since the number $\Omega_{m}\left(\mathscr{L}_{2}\right)$ lies inside the field $\mathbb{Q}\left(\mu_{p^{n}}\right)$, we can carry out the calculation inside the complex numbers. 
If $m=0$, then

$$
\begin{aligned}
\Omega_{0}\left(\mathscr{L}_{2}\right) & =p^{-n} \sum_{\alpha \in \mu_{p^{n}}} \frac{1}{\alpha^{-1}+1}=p^{-n} \sum_{\alpha \in \mu_{p^{n}}} \frac{1+\alpha}{2+2 \operatorname{Re}(\alpha)} \\
& =p^{-n} \sum_{\alpha \in \mu_{p^{n}}} \frac{(1+\operatorname{Re}(\alpha))+i \operatorname{Im}(\alpha)}{2+2 \operatorname{Re}(\alpha)}=\frac{1}{2 p^{n}} \sum_{\alpha \in \mu_{p^{n}}} 1+i \frac{\operatorname{Im}(\alpha)}{1+\operatorname{Re}(\alpha)} .
\end{aligned}
$$

However, for any $\alpha \in \mu_{p^{n}}$, we know that

$$
\frac{\operatorname{Im}\left(\alpha^{-1}\right)}{1+\operatorname{Re}\left(\alpha^{-1}\right)}=-\frac{\operatorname{Im}(\alpha)}{1+\operatorname{Re}(\alpha)},
$$

so the right-most part contributes nothing. We are left with the outcome that $\Omega_{0}\left(\mathscr{L}_{2}\right)$ equals $1 / 2$.

If $1 \leq m \leq p^{n}-1$ so $m \not \equiv 0\left(\bmod p^{n}\right)$, then

$$
\begin{aligned}
\Omega_{m}\left(\mathscr{L}_{2}\right) & =p^{-n} \sum_{\alpha \in \mu_{\rho^{n}}} \frac{\alpha^{m}}{\alpha^{-1}+1}=p^{-n} \sum_{\alpha \in \mu_{p^{n}}} \frac{\alpha^{m}\left(\left(\alpha^{-1}+1\right)-\alpha^{-1}\right)}{\alpha^{-1}+1} \\
& =p^{-n} \sum_{\alpha \in \mu_{p^{n}}} \alpha^{m}-\frac{\alpha^{m-1}}{\alpha^{-1}+1}=0-\Omega_{m-1}\left(\mathscr{L}_{2}\right) .
\end{aligned}
$$

By our inductive hypothesis, $\Omega_{m-1}\left(\mathscr{L}_{2}\right)=(-1)^{m-1} / 2$, which must then imply that $\Omega_{m}\left(\mathscr{L}_{2}\right)=(-1)^{m} / 2$.

The proof is finished.

REMARKS 3.3. (a) Theorem 1.1 is especially easy to implement on a computer. To approximate the generalised Bernoulli distribution using Riemann sums is of complexity at least $O\left(p^{2 n}\right)$. Using our $p$-adic Dirichlet series instead, we need only perform $p^{n}$ summations to achieve accuracy modulo $p^{n} \mathbb{Z}_{p}$.

(b) Proposition 3.2 also works for more general power series that are of the form $\mathscr{L}_{c}(X)=1 / X-c /\left((1+X)^{c}-1\right)$ with $\operatorname{gcd}(c, p)=1$. Mysteriously, $c=2$ is the only value where we get a clean Dirichlet expansion for the zeta-function; if $c>2$ then the coefficients are a mess. The $p$-adic $L$-function corresponds to a measure on the maximal real subfield of the $p$-cyclotomic extension of the rationals-it is surely no coincidence that when $c=\left[\mathbb{Q}\left(\mu_{p^{\infty}}\right): \mathbb{R} \cap \mathbb{Q}\left(\mu_{p^{\infty}}\right)\right]$, the coefficients are so simple.

(c) It is intriguing to ask whether $p$-adic Shintani zeta-functions, or the various $p$-adic $L$-functions attached to modular elliptic curves, have similar expansions as Dirichlet series. If the answer is in the affirmative, then these objects could be studied using techniques from analytic number theory. In order to answer these questions, we need to define the fractional derivative in terms of toroidal group schemes, and also for Lubin-Tate formal groups. 


\section{Acknowledgements}

The author thanks Mark Searle and Justin Kitt for many long discussions about the fractional calculus. He is also grateful to John Cremona and Paul Smith for verifying the formulae on a SUN Workstation.

\section{References}

[1] J. Coates and A. Wiles, 'On p-adic L-functions and elliptic units', J. Aust. Math. Soc. 26 (1978), $1-25$.

[2] R. Coleman, 'Dilogarithms, regulators and $p$-adic $L$-functions', Invent. Math. 69 (1982), 171-208.

[3] V. Vladimirov, I. Volovich and E. Zelemov, p-Adic analysis and mathematical physics (World Scientific, Singapore, 1994).

[4] L. Washington, Introduction to cyclotomic fields, Graduate Texts in Mathematics 83, 2nd edition (Springer, New York, 1997).

Department of Mathematics

University Park

Nottingham

England NG7 2RD

e-mail: dd@maths.nott.ac.uk 